\title{
Bluff-body drag reduction by extremum-seeking control
}

\author{
J.F. Beaudoin ${ }^{\mathrm{a}, *}$, O. Cadot $^{\mathrm{b}}$, J.L. Aider ${ }^{\mathrm{c}}$, J.E. Wesfreid ${ }^{\mathrm{c}}$ \\ ${ }^{a}$ Department of Research and Innovation, PSA Peugeot-Citroën, 2 route de Gisy, 78943 Vélizy-Villacoublay, France \\ ${ }^{\mathrm{b}}$ Unité de Mécanique, Ecole Nationale Supérieure de Techniques Avancées, Chemin de laHunière, 91761 Palaiseau Cedex, France \\ ${ }^{\mathrm{c}}$ Physique et Mécanique des Milieux Hétérogènes, Ecole Supérieure de Physique et Chimie Industrielles \\ (PMMH UMR 7636-CNRS-ESPCI), 10 rue Vauquelin, 75231 Paris Cedex 5, France
}

An actuator is used to control the drag exerted on a bluff-body at large Reynolds number (typically $\operatorname{Re}=20000$ ). The geometry is similar to a backward-facing step whose separation point is modified using a rotating cylinder at the edge. The slow fluctuations of the total drag are directly measured by means of strain gauges. The size of the lowpressure region behind the body is decreased and the drag reduced: the faster the rotation of the cylinder the lower the drag. The goal of the control is for the system to find itself an optimal set point, defined as the lowest cost of global energy consumption of the system (drag reduction versus energy used by the actuator). For this purpose an extremumseeking control method is applied in order to deal with the large background noise due to turbulence. It consists in a synchronous detection of the response measured in the drag measurements to a modulation of the actuator. This experiment attests from the real efficiency for local active control to reduce autonomously the global energy consumption of a turbulent separated flow, taking into account drag and actuator's energy cost.

Keywords: Bluff body; Drag reduction; Control; Extremum-seeking control

\section{Introduction}

From a general point of view, closed-loop control is supposed to drive in real time a dynamical system on a predetermined trajectory in the phase space. In many practical situations, one can use feedback control to maintain a system autonomously on its optimal functional point. In the case of unstable or turbulent flows, feedback control requires fast real-time analysis on signals having high level of background noise and many technical and theoretical problems have to be overcome to achieve it experimentally. Actually closed loop flow control is bound to numerical or theoretical works (Bewley et al., 2001; Patnaik and Wei, 2002; Kim, 2003) and most of the experimental studies are devoted to open loop control (Greenblatt and Wygnanski, 2000; Munshi et al., 1997). As far as we know, experimental studies of reactive flow control are both rare and recent (Fujisawa and Nakabayashi, 2002; Zhang et al., 2004). There are many realistic and important situations where external conditions are likely to change: the incidence of a wing, modification of the free-stream velocity, the yaw angle of a bluff-body. This phenomenon happens on much longer time scales than those of the flow turbulent fluctuations and have a major influence on the global dynamics of the flow.

\footnotetext{
*Corresponding author.

E-mail address: jeanfrancois.beaudoin@mpsa.com (J.F. Beaudoin).
} 
The present experiment shows a successful feedback loop control, whose set point is defined by the minimum of the global energy consumption of the system. The feedback law ensures that the system finds autonomously this minimum, whatever the external wind conditions.

This paper is organized as follows. We first describe the experimental setup, the measurement technique and the actuator. We show how the cost function is measured and its gradient estimated in real-time analysis. We describe the extremum seeking control methods (Krstić et al., 1995) that leads to the adaptive control. Finally, we summarize our findings in the conclusion.

\section{Experimental set-up}

The geometry of the bluff-body consists in a quarter of a circular cylinder laying on the floor just before a square cylinder (Fig. 1). The height of the bluff-body is $h=20 \mathrm{~mm}$, and its span length $100 \mathrm{~mm}$. We use a small open windtunnel whose cross-section is $100 \mathrm{~mm} \times 100 \mathrm{~mm}$. The flow velocity $U_{0}$ upstream of the bluff-body is measured with a hot wire probe located in front of the body. The main velocity ranges from 2 to $12 \mathrm{~m} \mathrm{~s}^{-1}$, so that Reynolds number $\operatorname{Re}=U_{0} h / v$ ranges from 3500 to 20000 . The flow is similar to the backward-facing step, where the recirculation bubble is responsible for the strong pressure drag.

The control device is a rotating cylinder located at the edge of the backward-facing step (see Fig. 1). This classical actuator is based on ideas of "moving walls" to reduce relative velocities at the flow boundaries [see, for instance, Modi et al. (1991), Munshi et al. (1997), or Patnaik and Wei (2002)]. The radius of the cylinder $R=5 \mathrm{~mm}$ and the rotation frequency can reach $250 \mathrm{~Hz}$ corresponding to a tangential velocity, or injection velocity, $U_{\text {inj }}=R \Omega \approx 7.5 \mathrm{~m} / \mathrm{s}$. We define the injection parameter $\zeta$ as the ratio between tangential velocity and the free-stream velocity $\zeta=U_{\text {inj }} / U_{0} \approx 1$ to obtain significant effects of drag decrease. The rotation of the cylinder clearly delays the separation, leading to a smaller recirculation bubble on the base of the bluff-body (as shown on Fig. 2) and a smaller drag (Beaudoin, 2004).
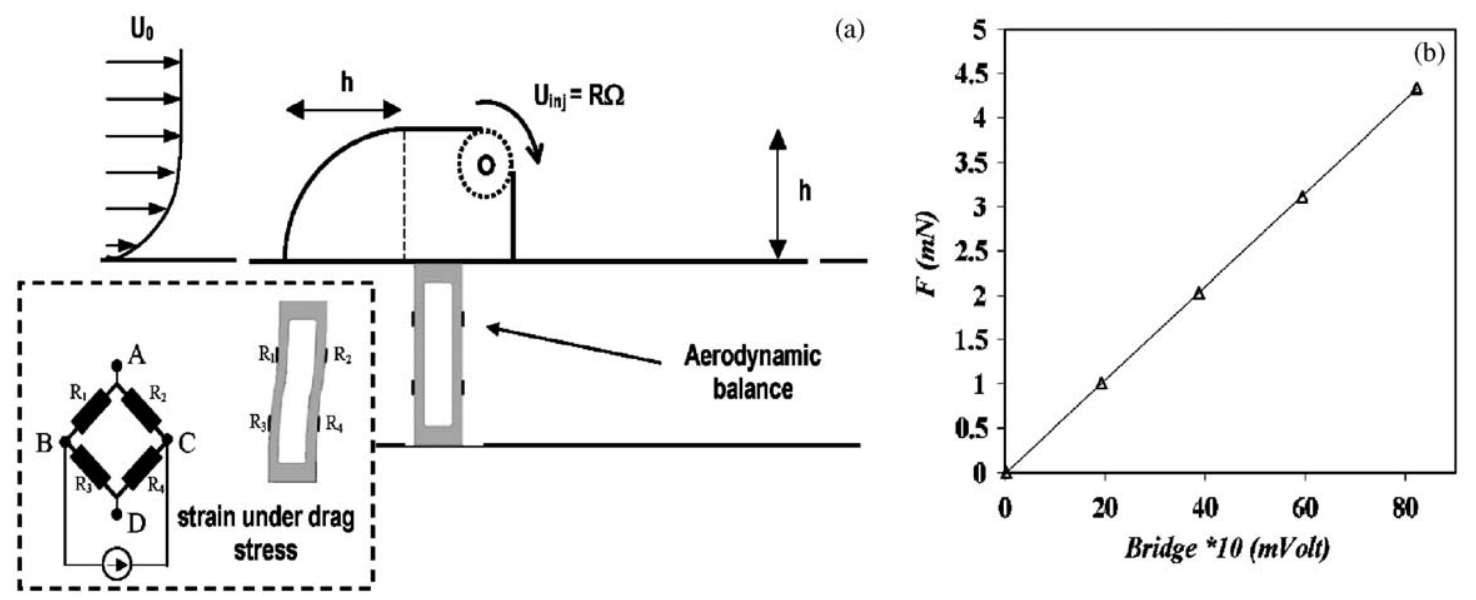

Fig. 1. (a) Sketch of the experimental set-up. A rotating cylinder located at the edge of the step modifies the characteristics of the flow separation. Inset: four strain gauges mounted in a full bridge configuration measure the global deformation and then the drag force. The output signal of the Vishay P3500 bridge is amplified by a factor 10 giving a sensitivity of $0.0527 \mathrm{~N} / \mathrm{V}$ say $0.527 \mathrm{~g} / \mathrm{mV}$ as displayed in (b) which is the calibration graph.
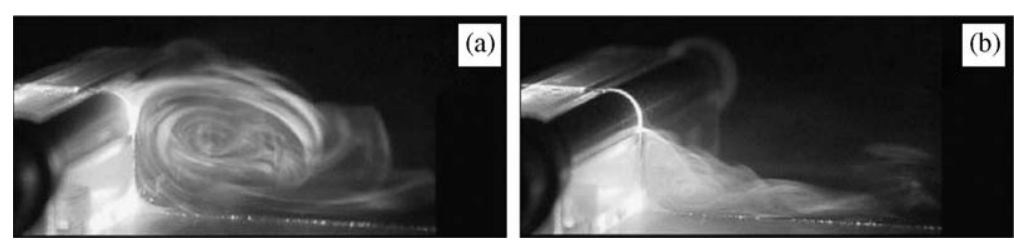

Fig. 2. Visualization of the separated flow downstream of the bluff-body for $U_{0}=2 \mathrm{~m} / \mathrm{s}$. (a) Without control; (b) with control $\left(U_{\text {inj }} / U_{0}=2\right)$. 
As we are interested in drag reduction, we chose to build a 1D aerodynamic balance to evaluate the drag of the bluffbody. We use a bimetallic brass strip $0.3 \mathrm{~mm}$ thick whose deformation is measured with four gauges. The bluff-body is positioned on a plate fixed to the brass strips and free to move a few tenths of a millimeter. The flow induces through the drag force a small displacement of the plate which causes a small deformation of the brass strips (see their shapes under the stress due to the drag in insert of Fig. 1(a)). The four gauges are used as inputs for a full Wheatstone bridge configuration, model P3500 from Vishay. The resistance variations due to temperature change are compensated two per two and the bridge measurements is proportional to 4 times the strain on each gauge. The calibration of the balance is obtained when the system is vertical by measuring the voltage at the bridge output for different masses laying on the bluff-body. The law displayed in Fig. 1(b) is simply proportional as expected for small strain and gives the total force experienced by the bluff-body. The drag measurements have a very low frequency response. Actually, the frequency response is limited by the natural frequency of the balance which is about $10 \mathrm{~Hz}$. It is simply measured as the response to an impulse excitation of the balance with no flow.

\section{Cost function $J$}

By definition, active control requires energy to activate the control device. It is then essential that the actuation does not use more energy than the system gains thanks to the control: the power balance (difference between gain and loss of energy) must be positive. In our case we want the highest drag reduction to reduce the dissipation through aerodynamic power $\mathscr{P}_{a}=D U_{0}$. To reach this goal we have to use electric power $\mathscr{P}_{e}$ for the rotation of the cylinder. The aerodynamic power $\mathscr{P}_{a}$ depends on both the rotation speed $\Omega$ and of the free-stream velocity $U_{0}$.

The measurements of electric power clearly show that $\mathscr{P}_{e}$ is a quadratic function of $\Omega$ (Fig. 3(a)), so that we fitted the power with the law $\mathscr{P}_{e}=\alpha \Omega^{2}\left(\alpha=4.44 \times 10^{-8} \mathrm{~W} /\left(\mathrm{rad} \mathrm{s}^{-1}\right)^{2}\right)$. Moreover, we observed that the electric power does not depend on the flow velocity (aerodynamic friction being negligible compared solid friction). The global power function $J\left(\Omega, U_{0}\right)$ is the sum of electric and aerodynamic power;

$$
J\left(\Omega, U_{0}\right)=\mathscr{P}_{a}\left(\Omega, U_{0}\right)+\mathscr{P}_{e}(\Omega)=D\left(\Omega, U_{0}\right) U_{0}+\alpha \Omega^{2} .
$$

The $J$ function is computed in real time on a first PC using single-point acquisitions of the rotation frequency $\Omega$, the free-stream velocity $U_{0}$ and the drag measurement $D$ with a $200 \mathrm{~Hz}$ sampling frequency. In Fig. 3(b), we present each term of the budget that has been time-averaged while $U_{0}=12 \mathrm{~m} / \mathrm{s}$. The error bars correspond to the r.m.s. value. The electric power is not measured in real time but estimated from $\Omega$ with the fit law of Fig. 3(a). As we can see, the aerodynamic power decreases as $\Omega$ increases showing the efficiency of the actuator to reduce the drag. Actually the reduction is effective whatever the mean flow velocity, and the larger the reduction, the larger is the rotation frequency $\Omega$ (Beaudoin, 2004). However, the power demanded by the actuator increases as $\Omega$ increases. As a result, the total power consumption has a minimum that determines an optimal set point. The $J(\Omega)$ function must have a minimum for all freestream velocities. We should then be able to use an "extremum-seeking control" to help the system to find its minimum.
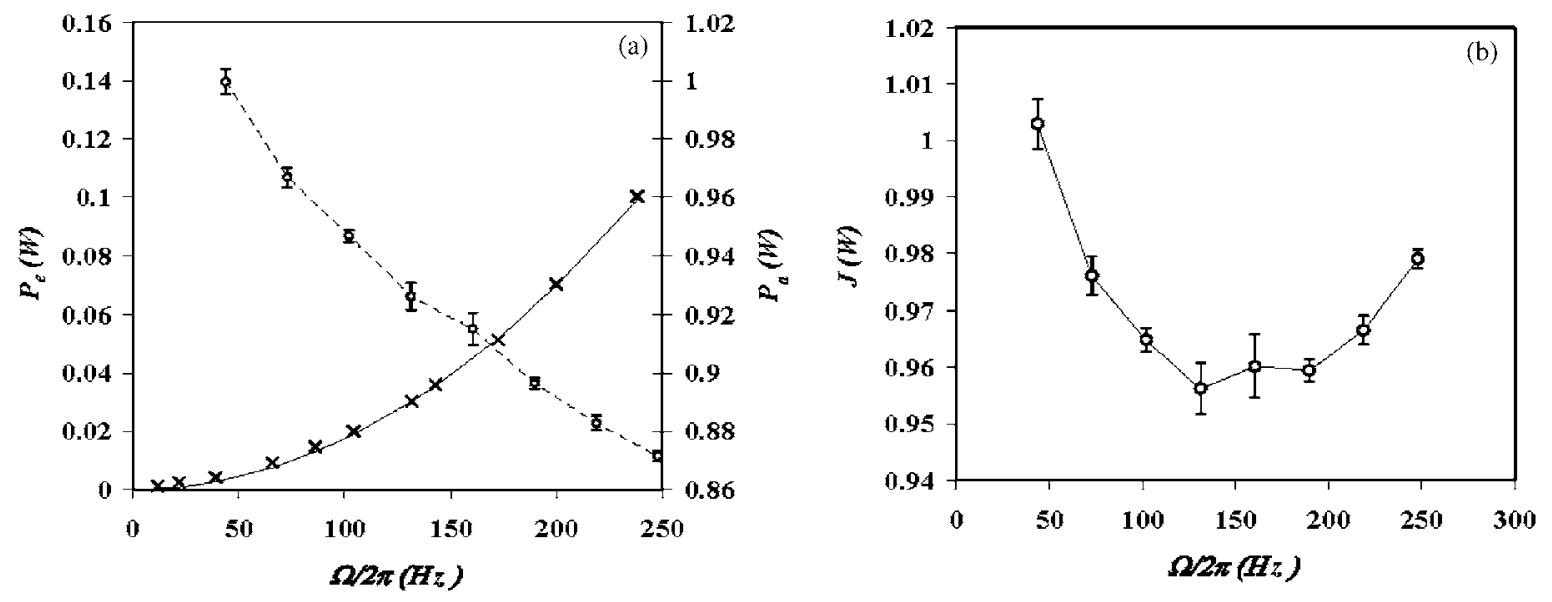

Fig. 3. (a) Measurements of the electrical power consumption $\mathscr{P}_{e}$ (crosses) fitted with a law $\mathscr{P}_{e}=\alpha \Omega^{2}$ (continuous line); aerodynamic power $\mathscr{P}_{a}$ with $U_{0}=12 \mathrm{~m} / \mathrm{s}$ (open circles); (b) $J\left(\Omega, U_{0}\right)$ as defined in Eq. (1). 


\section{4. $J$ gradient estimator: extremum-seeking control}

The control parameter $\Omega$ must be adjusted by the system to reach the state of minimum of the $J$ function. We choose to use an "extremum-seeking control" strategy. It consists in using a real-time gradient optimization method with the following feedback law:

$$
\frac{\mathrm{d} \Omega}{\mathrm{d} t}=-K \frac{\partial J}{\partial \Omega}
$$

where $K$ is a positive gain that is chosen by the experimentalist.

In the case of turbulent flows, the fluctuations of physical quantities are large, with a broad spectrum. It makes the evaluation of the gradient by classic finite difference nearly impossible. As proposed by Krstic and Wang (2000) we apply a sinusoidal perturbation method. It consists in modulating the input of our system in the following way:

$$
\Omega(t)=\Omega_{0}(t)+a \cos \left(2 \pi f_{m} t\right) .
$$

The constant $a$ has to be sufficiently large and $f_{m}$ lower than the cut-off frequency of the balance to detect the effect of the modulation on the drag measurements. We then took $a / 2 \pi=40 \mathrm{~Hz}$ and $f_{m}=1 \mathrm{~Hz}$. The objective is that $\Omega_{0}(t)$, which is a slowly varying function of time, reaches the value corresponding to the minimum of the $J$ function, whatever the free-stream velocity $U_{0}$. We will speak about modulated open-loop when the rotation is modulated and $\Omega_{0}$ constant in time.

To estimate the gradient of $(\partial J / \partial \Omega)\left(\Omega_{0}\right)$, another PC acquires $J(t)$ from the first PC as well as $\Omega(t)$ at a sampling rate of $50 \mathrm{~Hz}$ in a single-point acquisition mode. It then performs Fast Fourier Transform of both quantities over a sliding window of 4096 points corresponding to a $81.92 \mathrm{~s}$ time window. Both the amplitude $A_{J}\left(f_{m}\right)$ and the phase $\phi_{J}\left(f_{m}\right)$ of the mode $f_{m}$ are extracted from the sliding windows. The values are updated at a rate of $50 \mathrm{~Hz}$. Simultaneously, the same treatment is performed on $\Omega(t)$ in order to extract its phase $\phi_{\Omega}\left(f_{m}\right)$. The relationship between these measured quantities and the gradient is the following; the development of the $J$ function around the actual value $\Omega_{0}$ reads:

$$
J(\Omega)=J\left(\Omega_{0}\right)+\left(\Omega-\Omega_{0}\right) \frac{\partial J}{\partial \Omega} \cdot\left(\Omega_{0}\right) .
$$

Since $\Omega$ is modulated as in Eq. (3), the response of the $J$ function to the modulation can be modelled for $a$ small enough by

$$
J\left(\Omega_{0}+a \cos \left(2 \pi f_{m} t\right)\right)=J\left(\Omega_{0}\right)+a \cos \left(2 \pi f_{m} t\right) \frac{\partial J}{\partial \Omega}\left(\Omega_{0}\right),
$$

with this linearized expression, the $J$ and $\Omega$ functions are both harmonic. Their phase shift is 0 when $(\partial J / \partial \Omega)\left(\Omega_{0}\right)>0$, and $\pi$ when $(\partial J / \partial \Omega)\left(\Omega_{0}\right)<0$. This expression can be rewritten as

$$
J\left(\Omega_{0}+a \cos \left(2 \pi f_{m} t\right)\right)=J\left(\Omega_{0}\right)+a \cos \left(2 \pi f_{m} t+\varphi_{\pi}\right)\left\|\frac{\partial J}{\partial \Omega}\left(\Omega_{0}\right)\right\|,
$$

with $\varphi_{\pi}=0$ or $\pi$. Hence, the amplitude $A_{J}\left(f_{m}\right)$ of the Fourier mode of $J$ at the frequency $f_{m}$ gives $\left\|(\partial J / \partial \Omega)\left(\Omega_{0}\right)\right\|$, and its phase gives the sign of the gradient.

Experimentally, $J(t)$ is not a simple harmonic function (as considered in the previous linearized model), and $\varphi_{\pi}$ is not strictly equal to 0 or $\pi$. It is then important to measure $\varphi_{\pi}=\phi_{\Omega}\left(f_{m}\right)-\phi_{J}\left(f_{m}\right)$, which is the phase difference between both modes of $\Omega$ and $J$ at the frequency $f_{m}$, in order to obtain the sign of the gradient. As a result, the gradient is estimated as follows:

$$
\frac{\partial J}{\partial \Omega} \approx \frac{A_{J}\left(f_{m}\right)}{a} \operatorname{sign}\left(\cos \left(\phi_{\Omega}\left(f_{m}\right)-\phi_{J}\left(f_{m}\right)\right)\right) .
$$

Finally the second PC gives 50 orders per second to the motor controlling the rotation of the cylinder according to the following relation:

$$
\begin{aligned}
& \Omega_{n+1}=\Omega_{0, n+1}+a \cos \left(2 \pi f_{m}(n+1) \Delta t\right), \\
& \Omega_{0, n+1}=\Omega_{0, n}-K \frac{A_{J}\left(f_{m}\right)}{a} \operatorname{sign}\left(\cos \left(\phi_{\Omega}\left(f_{m}\right)-\phi_{J}\left(f_{m}\right)\right)\right) \Delta t .
\end{aligned}
$$



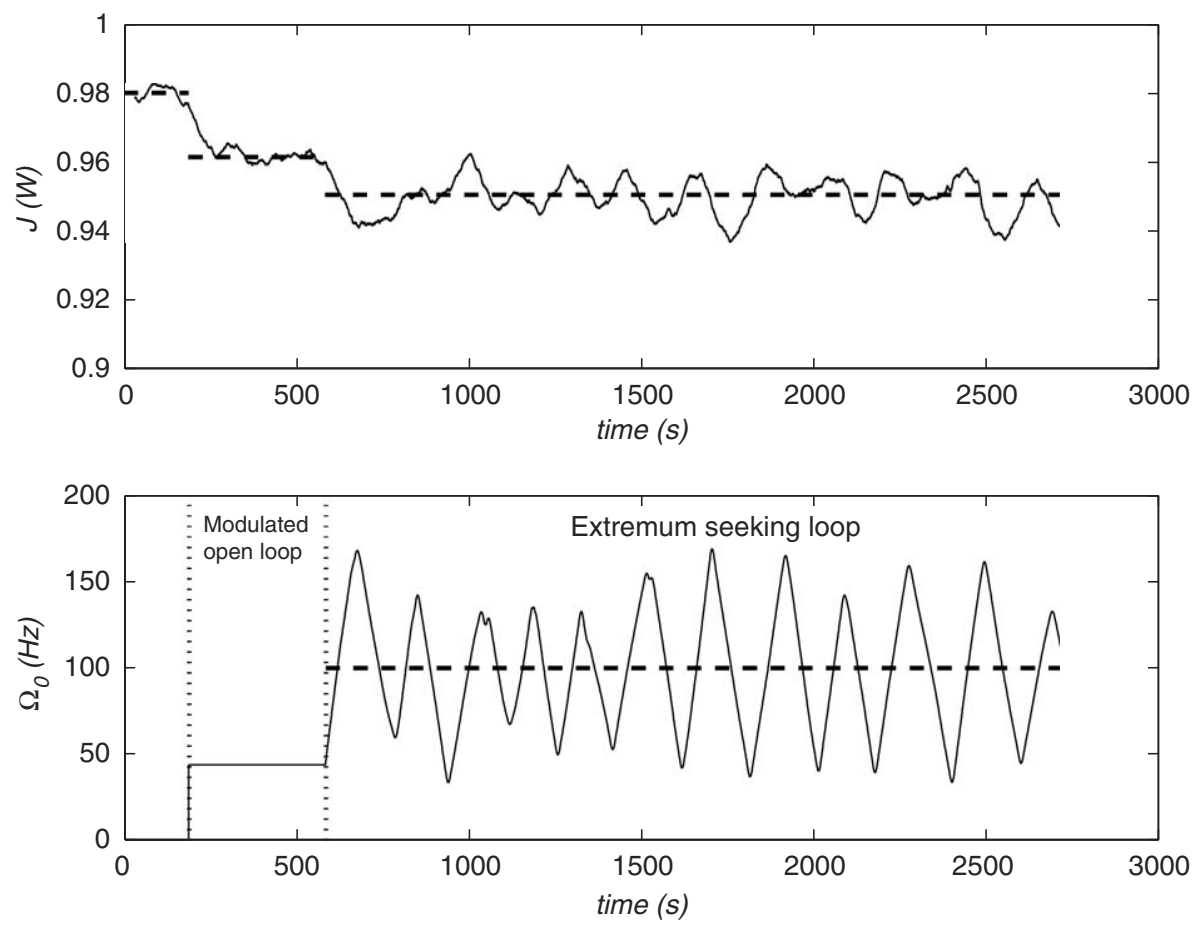

Fig. 4. Open-loop and closed-loop control experiment for $U_{0}=12 \mathrm{~m} / \mathrm{s}$. Upper figure: the fine continous line represents $J$, with the plateau of $J$ in dashed line. Lower: rotation $\Omega_{0}$ in continuous line, plateau of $\Omega_{0}$ in dashed line.

\section{Closed loop control}

At the beginning of the experiment, there is no forcing $(\Omega=0)$ and $J=J_{\text {ref }} \simeq 0.98 \mathrm{~W}$. After sometime, we apply the modulated open-loop control with $\Omega_{0}=45 \mathrm{~Hz}$ and observe the decreasing (in less than $80 \mathrm{~s}$ ) of the $J$ function toward a value close to $0.96 \mathrm{~W}$, which corresponds to the mean value we measured in open-loop control for $\Omega_{0}=45 \mathrm{~Hz}$ (Fig. 4). Then we trigger the closed-loop control and observe a new decrease of $J$ which finally begins to oscillate around a mean value close to $0.95 \mathrm{~W}$. During this last step, the system is completely autonomous and no longer needs any external intervention. The mean value of the rotation $\Omega_{0}$ increases as soon as the loop is closed and it oscillates around $100 \mathrm{~Hz}$. One can underline that the mean value $J$ corresponding to $\Omega_{0}=100 \mathrm{~Hz}$ in the open-loop experiment is about $0.95 \mathrm{~W}$, corresponding to the second plateau of the $J$ function (Fig. 4). The large fluctuations of $\Omega_{0}$ can be explained by the fact that the minimum of the $J$ function is not well defined.

\section{Concluding remarks}

We proposed a real-time gradient method using a sinusoidal modulation of the actuator. Thanks to the estimation of the gradient, the algorithm modifies the actuator mean rotation so that the system reaches and oscillates around its optimal state of lower energy consumption. From an academic point of view, we emphasize the fact that not many experiments in fluid mechanics attest from real efficiency for active control to globally reduce the energy consumption of a system. In this sense our experiments provide a very convincing illustration. Now we investigate the ability of the system to react to abrupt changes in flow conditions. These recent results are very encouraging and will be published soon.

\section{References}

Beaudoin, J.F., 2004. Contrôle actif d'écoulement en aérodynamique automobile. Ph.D. Thesis, Ecole des Mines de Paris.

Bewley, T.R., Moin, P., Temam, R., 2001. DNS-based predictive control of turbulence: an optimal benchmark for feedback algorithms. Journal of Fluid Mechanics 447, 179-225. 
Fujisawa, N., Nakabayashi, T., 2002. Neural network control of vortex shedding from a circular cylinder using rotational feedback oscillations. Journal of Fluids and Structures 16, 113-119.

Greenblatt, D., Wygnanski, I.J., 2000. The control of flow separation by periodic excitation. Progress in Aerospace Sciences 36, 487-545.

Kim, J., 2003. Control of turbulent boundary layers. Physics of Fluids 15, 1093-1105.

Krstić, M., Kanellakopoulos, I., Kokotovic, P.V., 1995. Nonlinear and Adaptive Control Design. Wiley, New-York.

Krstić, M., Wang, H.H., 2000. Stability of extremum seeking feedback for general nonlinear dynamic systems. Automatica 36, 595-601.

Modi, V.J., Mokhtarian, F., Fernando, M.S.U.K., Yokomizo, T., 1991. Moving surface boundary-layer control as applied to twodimensional air-foils. Journal of Aircraft 28, 104-112.

Munshi, S.R., Modi, V.J., Yokomizo, T., 1997. Aerodynamics and dynamics of rectangular prisms with momentum injection. Journal of Fluids and Structures 11, 873-892.

Patnaik, B.S.V., Wei, G.W., 2002. Controlling wake turbulence. Physical Review Letters 88, 554502.

Zhang, M.M., Cheng, L., Zhou, Y., 2004. Closed-loop-controlled vortex shedding and vibration of a flexibly supported square cylinder under different schemes. Physics of Fluids 16, 1439-1448. 